\title{
ANÁLISE GRÁFICA DO COMPORTAMENTO DE DIVERSAS SEQUÊNCIAS NUMÉRICAS ATRAVÉS DE SUAS EQUAÇÕES MATEMÁTICAS DE REGRESSÃO: UM PROCESSO DE ENSINO E APRENDIZAGEM DE MATEMÁTICA EM UM AMBIENTE COMPUTACIONAL
}

\author{
GRAPHICAL ANALYSIS OF THE BEHAVIOR OF VARIOUS NUMERICAL \\ SEQUENCES THROUGH THEIR MATHEMATICAL REGRESSION EQUATIONS: A \\ TEACHING AND LEARNING PROCESS OF MATHEMATICS IN A \\ COMPUTATIONAL ENVIRONMENT
}

\author{
Edel Alexandre Silva Pontes ${ }^{1}$ \\ Elinelson Gomes de Oliveira ${ }^{2}$ \\ Bruno Henrique Macêdo dos Santos Silva ${ }^{3}$
}

RESUMO: Na contemporaneidade é cada vez maior a necessidade de desenvolver meios na prática educacional de modo que a teoria estudada esteja diretamente correlacionada com os instrumentos do século XXI. A transformação tecnológica no mundo moderno exige que a escola esteja preparada para novos desafios no processo educativo e principalmente na metodologia de ensino e aprendizagem de matemática. Este trabalho investiga o comportamento de algumas sequências numéricas matemáticas conhecidas através de um estudo de regressão utilizando Linhas de Tendência geradas pelo software Excel 2003. Metodologicamente, o trabalho consiste em gerar um conjunto de dados que possa analisar o comportamento das sequências numéricas estabelecendo uma melhor equação matemática de ajuste. A contribuição deste trabalho é bastante significativa para a área de tecnologia educacional e no processo de ensino e aprendizagem de matemática, pois relaciona sequências numéricas importantes da matemática com equações de regressão que melhor se aproxima do modelo ideal. Diante disto, espera-se que este trabalho possa gerar estímulos necessários para que outros pesquisadores possam buscar práticas educacionais que fortaleçam o processo de ensino e aprendizagem de matemática com o propósito de gerar conhecimentos e saberes.

Palavras-chave: Ensino e aprendizagem de matemática; Sequências numéricas. Linhas de tendência.

\footnotetext{
${ }^{1}$ Doutor em Ciências da Educação com ênfase no Ensino de Matemática pela Universidad Tecnológica Intercontinental (UTIC). Professor do Programa de Pós-Graduação do Mestrado Profissional em Educação Profissional e Tecnológica (ProfEPT/IFAL).

${ }^{2}$ Mestre em Matemática pela Universidade Federal de Alagoas (UFAL). Professor do Instituto Federal de Alagoas - Campus Rio Largo.

${ }^{3}$ Licenciando em Matemática pela Universidade Federal de Alagoas (UFAL)
} 
ABSTRACT: Nowadays, there is an increasing need to develop means in educational practice so that the theory studied is directly correlated with the instruments of the 2ist century. Technological transformation in the modern world requires that the school be prepared for new challenges in the educational process and especially in the teaching and learning methodology of mathematics. This work investigates the behavior of some mathematical numerical sequences known through a regression study using Trend Lines generated by the Excel 2003 software. Methodologically, the work consists of generating a set of data that can analyze the behavior of the numerical sequences establishing a better equation. fit math. The contribution of this work is quite significant for the area of educational technology and in the process of teaching and learning mathematics, as it relates important numerical sequences in mathematics with regression equations that best approximates the ideal model. In view of this, it is hoped that this work can generate necessary stimuli so that other researchers can seek educational practices that strengthen the teaching and learning process of mathematics with the purpose of generating knowledge and knowledge.

Keywords: Mathematics teaching and learning. Numerical sequences. Trend lines.

\section{INTRODUÇÃO}

Nos dias atuais e diante do advento do computador, o uso das Tecnologias da Informação e Comunicação - TICs, no ambiente escolar, tem tornado uma possibilidade bastante usual no processo de ensino e aprendizagem de matemática e áreas afins. A utilização de softwares educativos matemáticos é fundamental no processo ensino e aprendizagem, pois permite que o aluno experimente construções de gráficos e descubra conceitos computacionalmente. Para Nascimento e Schimiguel (2017) existe um grande descompasso entre a geração que ensina e a geração que aprende, devido a toda transformação tecnológica do mundo moderno, surgindo assim mudança no processo ensino e aprendizagem.

Quando se propõe refletir sobre as novas formas de gerar e dominar o conhecimento, sobre as novas formas de explicações do real, nesse novo cenário tecnológico, que se delineia com o advento da Tecnologia, faz-se necessário traçar um paralelo entre a sociedade e o indivíduo, e contextualizar esse paralelo na nova cultura profissional que se estabelece com a disseminação da tecnologia. Com as novas tecnologias quais seriam as características e necessidades que essa nova cultura profissional informatizada exige dos indivíduos? (MISKULIN, 2003, p.218)

O novo modelo da sociedade baseia-se na comunicação e informação. Essa nova era tecnológica requer uma profunda revisão no sistema educativo e a escola tem um papel crucial na construção de modelos que possam aproximar os que ensinam daqueles que aprendem, e vice-versa. Os recursos tecnológicos exercem uma forte influência no processo de ensino e aprendizagem de matemática e essa "nova" escola exige do professor uma nova prática pedagógica, na qual ele possa ter a oportunidade de experimentar o computador, por meios de softwares educativos, em prol de um ensino voltado para uma realidade mais próxima do aluno aprendiz, possibilitando, cada vez 
mais, a construção de ideias, conceitos e procedimentos Nesta narração dinâmica, a educação torna-se um processo contínuo e aberto a novas possibilidades e desafios, através de um eixo de ligação entre a escola e a sociedade.

Percebe-se que reinventar o processo de ensino e aprendizagem de matemática é uma forma motivadora de gerar indivíduos prontos para enfrentar os desafios do mundo contemporâneo. As novas metodologias de ensino devem está associadas ao cotidiano dos aprendizes, pois, desta forma, acredita-se que minimizaremos as distâncias entre a teoria e a prática educacional (PONTES, et al., 2017, p.474)

Diversos pesquisadores de Educação Matemática estão em constante busca por novos caminhos para uma transformação científica da pedagogia que possa oferecer condições de tornar o ensino de matemática mais eficiente, fortemente programado, extremamente motivador e direcionado a habilidades, particularmente com o uso do computador. Segundo Ponte (1995) o principal motivo do insucesso do aluno no ensino de matemática resulta de seus modelos complexos, tornando-a difícil de entender. Muitos alunos acabam interiorizando, desde cedo, uma autoimagem de incapacidade frente à disciplina. Faz-se necessário que a disciplina de matemática seja exploratória, permitindo ao aluno pesquisar uma diversidade de aplicações com dados reais ou exemplos práticos que possa tornar os processos matemáticos mais próximos de sua realidade.

O atual ensino de matemática não possibilita a cada indivíduo conseguir seu potencial criativo. Um dos principais problemas $\mathrm{da}$ Educação atualmente é o baixo índice de utilização da tecnologia no processo Educacional. A incorporação de toda tecnologia disponível no mundo é essencial para tornar a escola uma instituição que ofereça ensino de qualidade. Estamos próximos do novo milênio, bem antes de sua chegada já presenciamos um grande progresso intelectual, e também um progresso tecnológico que alcança estágios altíssimos de desenvolvimento e complexidade, mais do que nunca, o domínio de conhecimentos de informática aplicados à matemática se faz necessário para que possamos acompanhar a evolução da época (ALBUQUERQUE, 200o, p.2)

A escola sofre a pressão de uma sociedade que exige drástica transformação em seu sistema educacional e tem na matemática computacional um instrumento de geração de novos conhecimentos e saberes, apoiado pelas rápidas mudanças no mundo globalizado. A utilização de modelos computacionais na escola básica e a popularização da matemática, em especial, através dos projetos de olimpíadas vêm caracterizando-se como práticas imprescindíveis no processo de ensino e aprendizagem de matemática. De acordo com Tenório, et al. (2017) o uso de Softwares educativos matemáticos são extremamente importantes para uma melhor performance do aprendiz na escola que frequenta, pois permite que ele experimente construções e exponha conceitos a partir do manuseio de gráficos, tabelas e medidas. 
A sala de aula se torna ambiente agradável quando se apresenta práticas motivadoras e criativas com perfeita sintonia com o mundo moderno, recheado de indivíduos de raciocínio lógico apurado, intuitivos $e$ de pensamento matemático aumentado. $O$ mundo tecnológico e a matemática se confundem por haver uma relação biunívoca entre o modelo criador e o ser criado. O ensino tradicional, dos conteúdos de matemática, deve ser substituído por um ensino motivador aproximando o aluno da sua realidade, essas mudanças devem-se essencialmente ao surgimento da era computacional (PONTES, 2019, p.3).

Este artigo objetivou: (i) investigar o comportamento de algumas sequências numéricas matemáticas conhecidas através de um estudo de regressão utilizando Linhas de Tendência geradas pelo software Excel 2003; (ii) Encontrar a equação matemática de regressão que melhor se ajuste a cada sequência numérica; e (iii) Estimular o processo de ensino e aprendizagem de matemática através de um ambiente computacional.

Entende-se que atividades desta natureza fortalecerá o processo de ensino e aprendizagem do conteúdo, como também aproximará o modelo abstrato da prática concreta. Percebe-se que dois pontos são fundamentais para o entendimento do trabalho: I. As sequências numéricas matemáticas: número primo, sequência de Fibonacci, sucessão de Farey, número hexagonal, número de Catalan, Progressão Aritmética, Progressão Geométrica e quadrados perfeitos; e II. As linhas de tendência associadas as sua equações matemáticas através da ferramenta Regressão do Software Excel: linear, exponencial, logarítmica e potência.

Toda proposta que envolve Tecnologias da Informação e Comunicação - TICs no espaço educacional são relevantes e geram no aluno aprendiz maior interesse e motivação. Desta forma, este trabalho justifica-se pelo motivo de sugerir uma proposta de ensino e aprendizagem de matemática atrelada a sequências numéricas conhecidas e modelos de regressão, através de um ambiente computacional.

\section{FUNDAMENTAÇÃO TEÓRICA}

No contexto contemporâneo, o processo ensino e aprendizagem de matemática através do uso de tecnologias educacionais são do ponto de vista temporal bastante recente. Segundo os Parâmetros Curriculares Nacionais (BRASIL, I998), as experiências efetivas do computador na escola têm mostrado uma maior aproximação do professor com o aluno. A forte presença do computador nas bancas escolares transformou significativamente a prática pedagógica dos professores de matemática e o método de aprendizagem do aluno da educação básica.

A mudança de cenário da sala de aula para um ambiente computacional, com a participação do aluno, é crucial na construção de conceitos a partir de investigações para, posteriormente, apropriar-se da teoria (PAULA et al., 2016)

[...] é preciso que o professor dê novo significado a sua prática de sala de aula por meio da mediação que deve considerar três aspectos: o processo tecnológico, o processo pedagógico e o processo formativo. $\mathrm{O}$ primeiro refere-se às potencialidades encontradas na tecnologia que 
será utilizada. $\mathrm{O}$ processo pedagógico está relacionado à maneira que as atividades são desenvolvidas e que objetivos querem ser alcançados. Por fim, o formativo é o processo de desenvolvimento da atividade e inclui a recriação e redefinição dos procedimentos de uso dos instrumentos utilizados (CARNEIRO \& PASSOS, 2014, p.I04)

Diante desta nova possibilidade educacional, através da proposta da utilização de tecnologia na educação, observa-se uma alteração no modo de ensinar e de aprender matemática. $\mathrm{O}$ ambiente computacional é propicio para minimizar as dificuldades encontradas pelos "alunos tecnológicos" na aprendizagem de matemática. Muitas vezes, essas dificuldades são geradas pela falta de motivação destes "alunos tecnológicos" em acompanhar um ensino de matemática fundamentada na visão tradicional de repassar conteúdos sem uma aplicação prática e real do modelo.

Para Pontes et al. (2021), a prática escolar e a base teórica no processo de ensino e aprendizagem de matemática devem se conectar de modo indefinido para minimizar as discrepâncias entre o que se recomenda a ensinar, encargo do educador, e o que se capacita a aprender, valores para o aluno.

$\mathrm{O}$ ensino e aprendizagem de matemática é um processo construtivo e organizado, na qual acontece a partir da interação do homem com o meio em que vive, e não apenas pela obrigatoriedade da transmissão e recepção de informações. "O educador deve estar preparado para compreender e acompanhar com destreza a nova geração de alunos tecnológicos" (PONTES, 2013, p.2).

Este artigo propõe, em seus objetivos, relacionar sequências numéricas conhecidas com métodos de regressão (linhas de tendência), através do software Excel, e para isso acontecer é necessário definirmos alguns conceitos e relações matemáticas indispensáveis no estudo proposto. Nosso estudo se dedicará a famosas sequências numéricas: número primo, sequência de Fibonacci, sucessão de Farey, número hexagonal, número de Catalan, Progressão Aritmética, Progressão Geométrica e quadrados perfeitos.

A definição matemática de sequência ou sucessão é uma função que associa para cada $n$ do domínio um valor no conjunto imagem $f(n)$. O tamanho de uma sucessão é o número que está sequência possui, podendo se ter sequências finitas ou infinitas.

$\mathrm{O}$ número primo $n$ é um número inteiro que tem somente quatro divisores distintos, -I, I e $-n, n$. O Teorema Fundamental da Aritmética afirma que qualquer número inteiro diferente de um, pode ser escrito de forma única como um produto de números primos. Os números primos são: 2, 3, 5, 7, II, I3, I7, 19, 23, 29,....

A sucessão de Fibonacci deve-se ao grande matemático da idade medieval Leonardo de Pisa (II75-1250) ou Leonardo de Fibonacci quando publicou a sua obra Liber Abaci, em 1202. A sucessão de Fibonacci é uma sequência matemática que é definida recursivamente pela seguinte fórmula:

$$
F_{n}=\left\{\begin{array}{c}
F_{1}=1 \\
F_{2}=1 \\
F_{n-1}+F_{n-2} \nabla n \geq 3, n \in \mathrm{N}
\end{array}\right.
$$

Então, a Sucessão de Fibonacci é dada por: I, I, 2, 3, 5, 8, 13, 21, 34, 55,... 
Uma sequência numérica de Farey é uma sucessão matemática de frações irredutíveis entre o e I que tem um denominador menor ou igual a $n$ em ordem crescente, sem repetições. A sequência de Farey está relacionada às equações Diofantinas. Qualquer sequência de Farey começa em o e termina no I. Por exemplo, a sequência de Farey para $n=5$, temos: $0, \frac{1}{5}, \frac{1}{4}, \frac{1}{3}, \frac{2}{5}, \frac{2}{4}, \frac{3}{5}, \frac{2}{3}, \frac{3}{4}, \frac{4}{5}, 1$.

Um número hexagonal é um número poligonal que está representado na forma de um hexágono. A construção de números hexagonais é gerada pela fórmula de recorrência:

$$
H_{n}=\frac{2 n(2 n-1)}{2}
$$

Os números hexagonais são: I, 6, I5, 28, 45, 66, 91, I20, 153, 190,... .

O número de Catalan foi desenvolvido pelo matemático belga Eugène Charles Catalan (1814-1894), tem origem na combinatória e formam uma sequência de números naturais. Os números de Catalan podem representar o número de maneiras de dividir um polígono de $n+2$ lados em $n$ triângulos. $\mathrm{O} n$ - ésimo número de Catalan é dado em termos de coeficientes binominais por:

$$
C_{n}=\frac{1}{n+1}\left(\begin{array}{c}
2 n \\
n
\end{array}\right) \text { para } n \geq 0
$$

Daí, os números de Catalan são: I, I, 2, 5, I4, 42, I32, 429, I430, 4862,...

Uma Progressão Aritmética (PA) é toda sequência numérica que a partir do segundo termo cada elemento é a soma de seu antecessor por uma constante $r$, chamada razão. Seja $\left(a_{1}, a_{2}, a_{3}, \ldots, a_{n}\right)$ uma PA com $n$ termos, então a fórmula do termo geral desta PA é dada por:

$$
a_{n=} a_{1}+(n-1) r
$$

Os números naturais ímpares positivos formam uma PA de razão dois: I, 3, 5, 7, $9, \mathrm{II}, \mathrm{I} 3, \mathrm{I}, \ldots .$.

Uma Progressão Geométrica (PG) é toda sequencia numérica que a partir do segundo termo cada elemento é o produto de seu antecessor por uma constante $q$, chamada razão. Seja $\left(a_{1}, a_{2}, a_{3}, \ldots, a_{n}\right)$ uma PG com $n$ termos, então a fórmula do termo geral desta PG é dada por:

$$
a_{n=} a_{1} q^{n-1}
$$

A sequência de todos os números positivos na forma $2^{n}$, com $n$ natural positivo, é uma PG de razão dois: $2,4,8,16,32,64,128,256$ 512,...

Um número $x$ é um quadrado perfeito, quando $x=n^{2}$ para todo $n$ inteiro positivo. Somente o número quadrado perfeito possui raiz quadrada exata.

Os quadrados perfeitos são: $\mathrm{I}, 2,4,9, \mathrm{I} 6,25,36,49,64,81, \ldots$.

A Linha de Tendência é um gráfico estatístico gerado por uma equação matemática que melhor se ajusta a um conjunto de dados $(x, y)$. No Excel essa ferramenta é chamada Regressão que pode gerar diversas Linhas de Tendência. "A utilização de um Software educacional para explicar modelos matemáticos tem uma importância fundamental para um melhor entendimento de conceitos extremamente necessários no processo de ensino e aprendizagem de matemática" (PONTES, et al. 
2018, p.777). Neste estudo usaremos as seguintes Linhas de Tendência associadas as suas respectivas equações:

Linha de Tendência Linear: $y=A x+B$

Linha de Tendência Exponencial: $y=B e^{A x}$

Linha de Tendência Logarítmica: $y=A \ln x+B$

Linha de Tendência Potência: $y=B x^{A}$

A Linha de Tendência Linear apresenta um comportamento que pode aumentar ou diminuir seguindo uma taxa fixa. A Linha de Tendência Exponencial segue um comportamento que seus valores estão aumentando (diminuindo) com taxas cada vez mais elevadas. A Linha de Tendência Logarítmica expressa um comportamento que sua taxa aumenta ou diminui rapidamente e logo depois nivela. A Linha de Tendência Potência é uma linha curva de maneira que seus valores aumentam a uma taxa específica.

Acompanhado da equação matemática de Regressão, o Excel também gera um fator R-quadrado, chamado coeficiente de correlação, que mostra, em porcentagem, o quanto a equação matemática de Regressão se aproxima do valor ideal, isto é, quanto mais próximo estiver de um, o R-quadrado, melhor o ajuste da equação ao conjunto de dados.

\section{MATERIAIS E METÓDOS}

Metodologicamente, o trabalho consiste em gerar um conjunto de dados $(x, y)$ que possa analisar o comportamento das sequências numéricas matemáticas estabelecendo uma melhor equação de ajuste. O Software utilizado foi a planilha do Excel 2003 em uma ferramenta chamada Regressão - Linhas de Tendência.

O conjunto de dados gerados $(x, y)$ será um conjunto de pontos, onde a variável $x$ representa a ordem de cada número da sequência numérica, e a variável $y$ representa os números da sequência numérica.

Inicialmente será gerado um conjunto de pontos $(x, y)$ para cada sequência definida, entre elas: número primo, sequência de Fibonacci, sucessão de Farey, número hexagonal, número de Catalan, Progressão Aritmética, Progressão Geométrica e quadrados perfeitos. Em seguida, para cada sequência, será construído, utilizando o Excel 2003, o gráfico de regressão com suas linhas de tendência (linear, exponencial, logarítmica e potência) e o cálculo do $\mathrm{R}$ de correlação. Finalmente, um parecer será apresentado com o melhor e o pior ajuste de regressão para cada sequência proposta.

\section{RESULTADOS E DISCUSSÕES}

A proposta do artigo é encontrar uma equação matemática que melhor se ajuste a uma sequência numérica. Através de uma análise gráfica de regressão por meio do Software Excel apresentamos nesta seção a resposta para as sequências sugeridas.

A Figura I mostra que a equação matemática que melhor se aproxima do modelo ideal da sequência de números primos (Tabela $\mathrm{I}$ ) é a Linha de Tendência Potência ( $\mathrm{R}$ quadrado=o,9795), como também, a Linha de Tendência Linear (R-quadrado=o,976r). 
Percebe-se que a Linha de Tendência Logarítmica tem o pior ajuste (Rquadrado=0,7943).

Tabela I: Conjuntos de pontos dos números primos

\begin{tabular}{lllllllllllll}
\hline $\mathbf{x}$ & I & 2 & 3 & 4 & 5 & 6 & 7 & 8 & 9 & IO & II & I2 \\
\hline $\mathbf{y}$ & 2 & 3 & 5 & 7 & II & I3 & I7 & I9 & 23 & 29 & 3 I & 37 \\
\hline
\end{tabular}

Fonte: elaboração dos autores.

Figura I: Linhas de Tendência - Números Primos

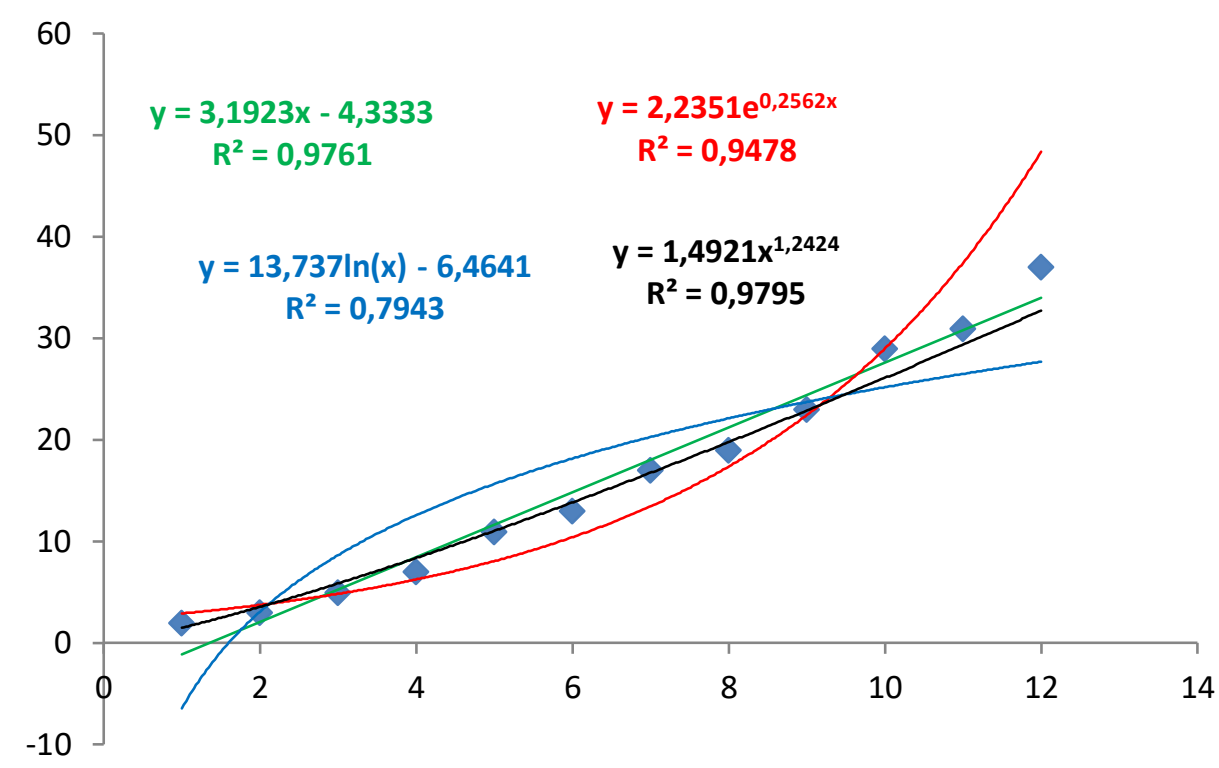

Fonte: elaboração dos autores (Excel).

A Figura 2 mostra que a equação matemática que melhor se aproxima do modelo ideal da sequência de Fibonacci (Tabela 2) é a Linha de Tendência Exponencial (Rquadrado=0,9963). Percebe-se também que a Linha de Tendência Logarítmica (Rquadrado=0,4377) e a Linha de Tendência Linear ( $\mathrm{R}$-quadrado=0,696) apresentaram os piores ajustes.

Tabela 2: Conjuntos de pontos da Sequência de Fibonacci

\begin{tabular}{lllllllllllll}
\hline $\mathbf{x}$ & I & 2 & 3 & 4 & 5 & 6 & 7 & 8 & 9 & IO & II & I2 \\
\hline $\mathbf{y}$ & I & I & 2 & 3 & 5 & 8 & I3 & 2 I & 34 & 55 & 89 & I44 \\
\hline
\end{tabular}

Fonte: elaboração dos autores. 
Figura 2: Linhas de Tendência - Sequência de Fibonacci

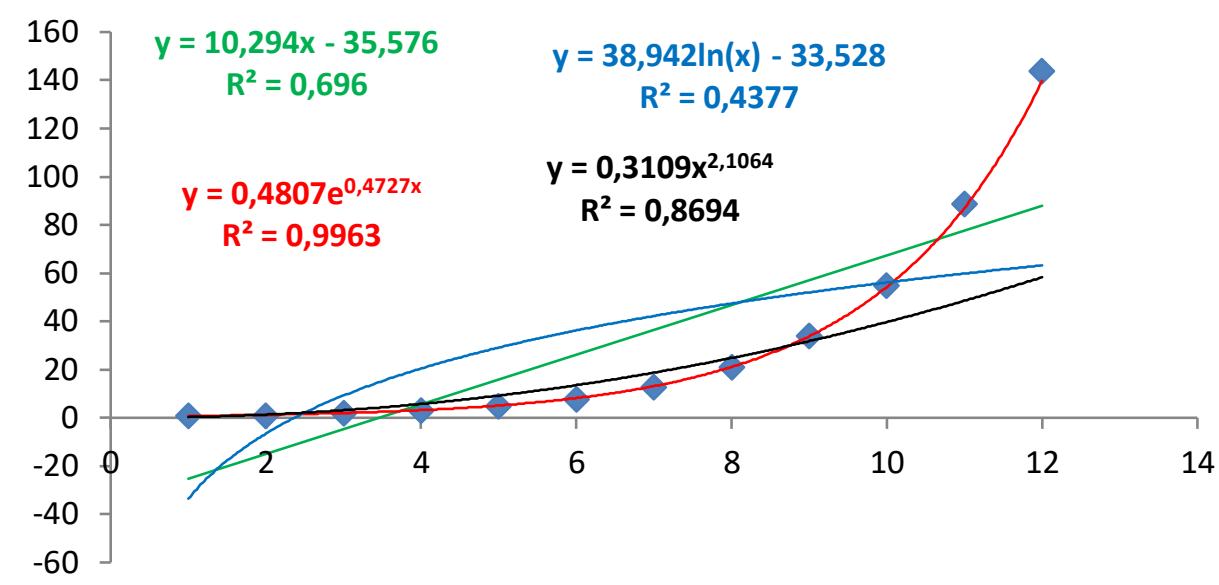

Fonte: elaboração dos autores (Excel).

A Figura 3 mostra que a equação matemática que melhor se aproxima do modelo ideal da sucessão de Farey (Tabela 3) é a Linha de Tendência Linear ( $R$ quadrado=o,9848), seguida da Linha de Tendência Logarítmica (R-quadrado=o,9069). Nota-se que a Linha de Tendência Exponencial (R-quadrado=0,4286) tem o pior ajuste.

Tabela 3: Conjuntos de pontos da Sucessão de Farey

\begin{tabular}{llllllllllll}
\hline $\mathbf{x}$ & $\mathrm{I}$ & 2 & 3 & 4 & 5 & 6 & 7 & 8 & 9 & IO & II \\
\hline $\mathbf{y}$ & $\mathrm{o}$ & $\mathrm{I} / 5$ & $\mathrm{I} / 4$ & $\mathrm{I} / 3$ & $2 / 5$ & $2 / 4$ & $3 / 5$ & $2 / 3$ & $3 / 4$ & $4 / 5$ & I \\
\hline
\end{tabular}

Fonte: elaboração dos autores.

Figura 3: Linhas de Tendência - Sucessão de Farey

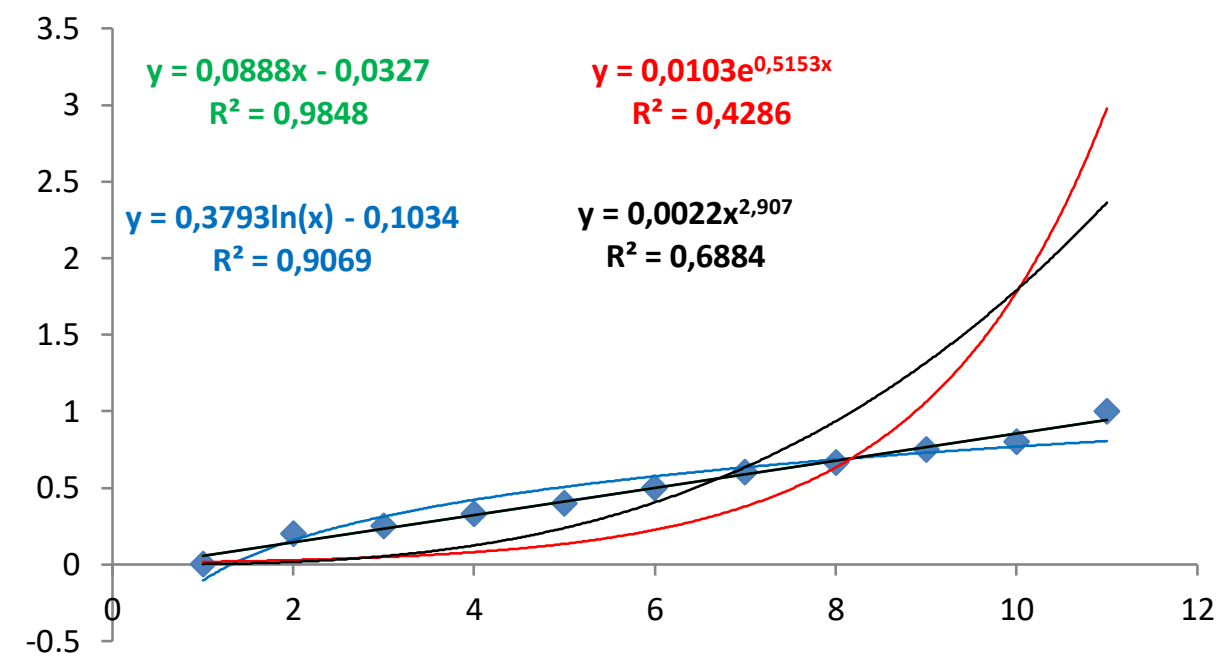

Fonte: elaboração dos autores (Excel). 
A Figura 4 mostra que a equação matemática que melhor se aproxima do modelo ideal do Número Hexagonal (Tabela 4) é a Linha de Tendência Potência (Rquadrado=0,9978), quase uma correlação perfeita. Percebe-se também que a Linha de Tendência Logarítmica ( $\mathrm{R}$-quadrado=0,7204) tem o pior ajuste.

Tabela 4: Conjuntos de pontos do Número Hexagonal

\begin{tabular}{|c|c|c|c|c|c|c|c|c|c|c|c|c|}
\hline $\mathbf{x}$ & I & 2 & 3 & 4 & 5 & 6 & 7 & 8 & 9 & IO & II & I2 \\
\hline$y$ & I & 6 & 15 & 28 & 45 & 66 & 9I & 120 & 153 & 190 & 231 & 276 \\
\hline
\end{tabular}

Fonte: elaboração dos autores.

Figura 4: Linhas de Tendência - Número Hexagonal

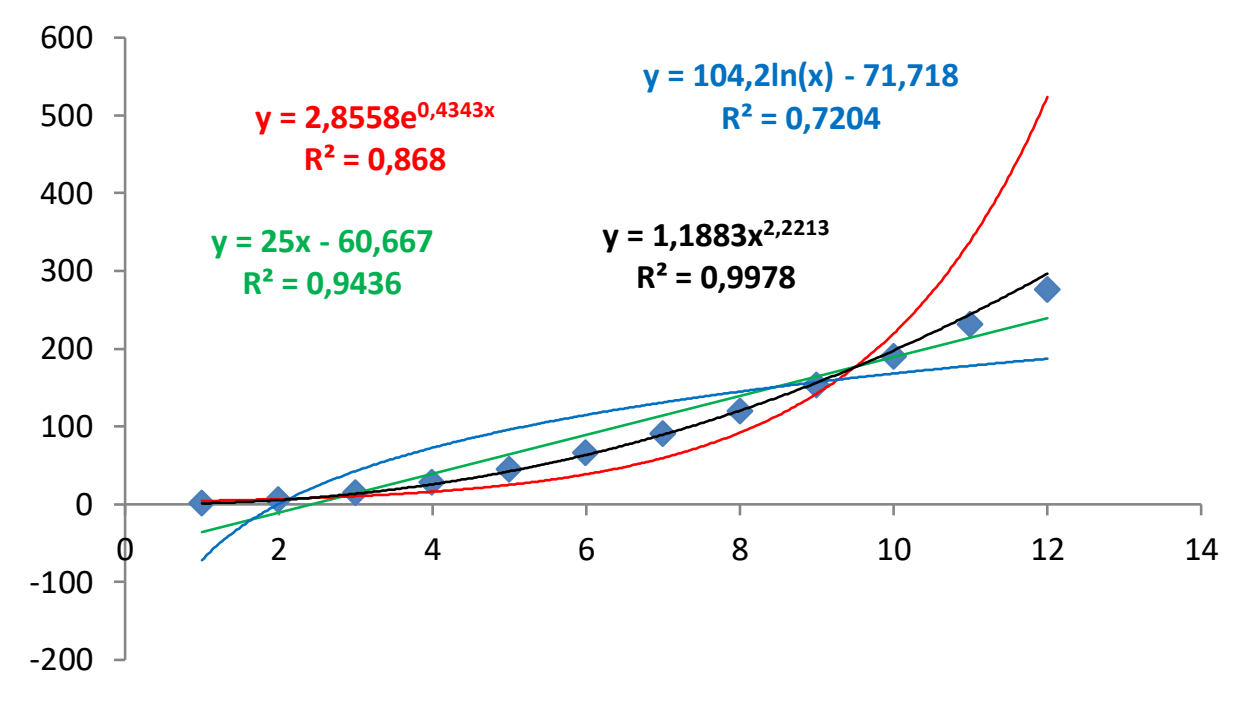

Fonte: elaboração dos autores (Excel).

A Figura 5 mostra que a equação matemática que melhor se aproxima do modelo ideal da sequência do Número de Catalan (Tabela 5) é a Linha de Tendência Exponencial (R- quadrado=0,9729). Nota-se que a Linha de Tendência Logarítmica (Rquadrado=0,2565) e a Linha de Tendência Linear ( $\mathrm{R}$-quadrado=0,4513) apresentaram os piores ajustes.

Tabela 5: Conjuntos de pontos do Número de Catalan

\begin{tabular}{ccccccccccc}
\hline $\mathbf{x}$ & $\mathrm{I}$ & $\mathbf{2}$ & 3 & 4 & 5 & 6 & 7 & 8 & 9 & I0 \\
\hline $\mathbf{y}$ & $\mathrm{I}$ & $\mathrm{I}$ & 5 & $\mathrm{I} 4$ & 42 & $\mathrm{I} 32$ & 429 & $\mathrm{I} 430$ & 4862 & I6796 \\
\hline
\end{tabular}

Fonte: elaboração dos autores. 
Figura 5: Linhas de Tendência - Número de Catalan

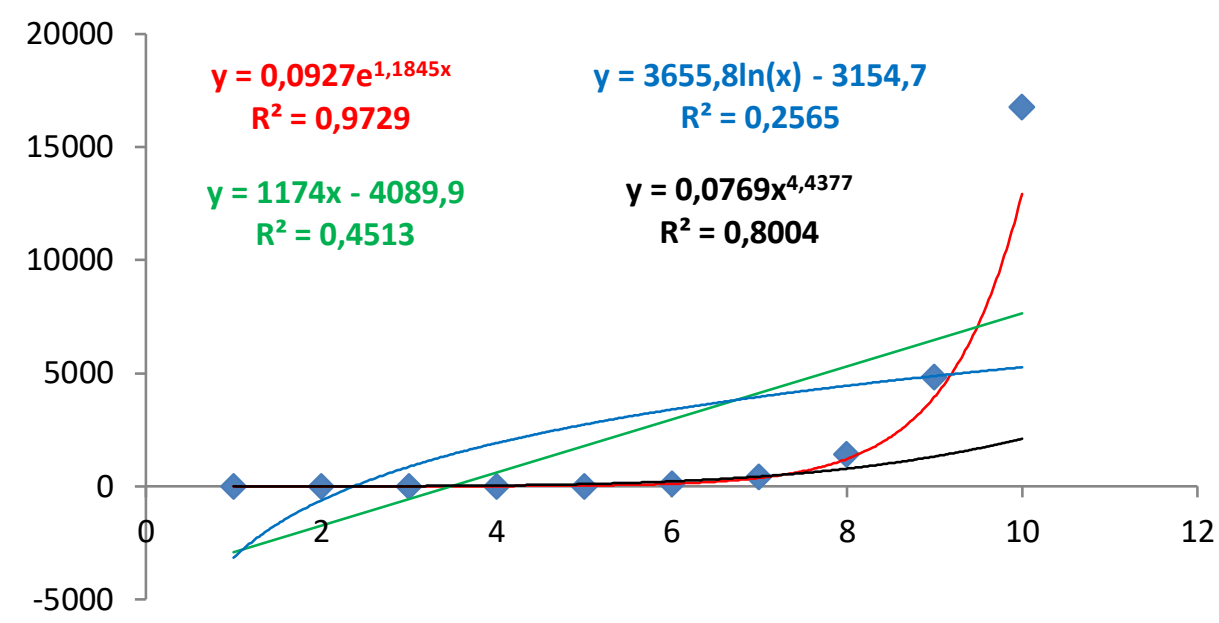

Fonte: elaboração dos autores (Excel).

A Figura 6 mostra que a equação matemática que melhor se aproxima do modelo ideal de uma PA de termo geral $a_{n}=2 n-1$ (Tabela 6) é a Linha de Tendência Linear $(R$ - quadrado= $\mathrm{I})$, correlação perfeita. Observa-se também que a Linha de Tendência Logarítmica (R-quadrado=o,895I) e a linha Tendência Exponencial (Rquadrado $=0,8434)$ tiveram os piores ajustes.

Tabela 6: Conjuntos de pontos de uma PA de termo geral $a_{n}=2 n-1$

\begin{tabular}{|c|c|c|c|c|c|c|c|c|c|c|c|c|}
\hline $\mathbf{x}$ & I & 2 & 3 & 4 & 5 & 6 & 7 & 8 & 9 & IO & II & I2 \\
\hline $\mathbf{y}$ & I & 3 & 5 & 7 & 9 & II & I3 & I5 & I7 & I9 & $2 I$ & 23 \\
\hline
\end{tabular}

Fonte: elaboração dos autores.

Figura 6: Linhas de Tendência - PA de termos geral $a_{n}=2 n-1$

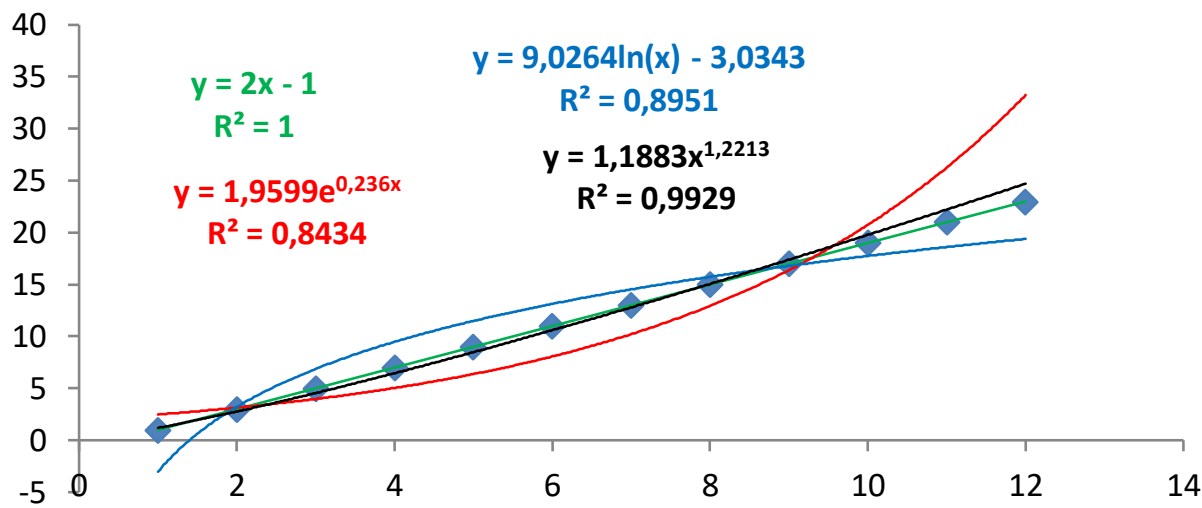

Fonte: elaboração dos autores (Excel). 
A Figura 7 mostra que a equação matemática que melhor se aproxima do modelo ideal de uma PG de termo geral $a_{n}=2^{n}$ (Tabela 7) é a Linha de Tendência Exponencial (R-quadrado=I), correlação perfeita. Observa-se que a Linha de Tendência Logarítmica (R-quadrado=0,3315) e a Linha de Tendência Linear ( $\mathrm{R}$-quadrado=0,5668) apresentaram os piores ajustes.

Tabela 7: Conjuntos de pontos de uma PG de termo geral $a_{n}=2^{n}$

\begin{tabular}{lllllllllllll}
\hline $\mathbf{x}$ & $\mathrm{I}$ & 2 & 3 & 4 & 5 & 6 & 7 & 8 & 9 & I0 & II & I2 \\
\hline $\mathbf{y}$ & 2 & 4 & 8 & $\mathrm{I} 6$ & 32 & 64 & $\mathrm{I2} 8$ & 256 & 512 & I024 & 2048 & 4096 \\
\hline
\end{tabular}

Fonte: elaboração dos autores.

Figura 7: Linhas de Tendência - PG de termo geral $a_{n}=2^{n}$

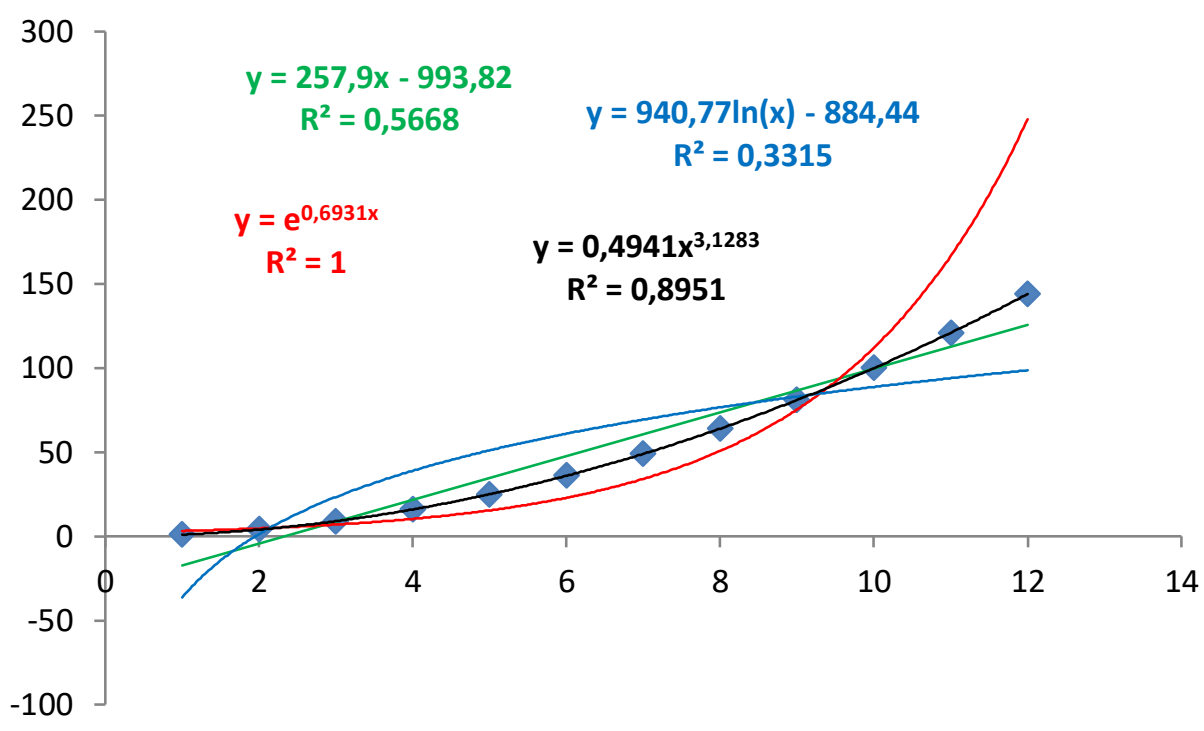

Fonte: elaboração dos autores (Excel).

A Figura 8 mostra que a equação matemática que melhor se aproxima do modelo ideal dos quadrados perfeitos (Tabela 8) é a Linha de Tendência Potência (Rquadrado=I), correlação perfeita. Observa-se que a Linha de Tendência Logarítmica (Rquadrado $=0,728 \mathrm{I}$ ) tem o pior ajuste.

Tabela 8: Conjuntos de pontos dos Quadrados Perfeitos

\begin{tabular}{lllllllllllll}
\hline $\mathbf{x}$ & $\mathrm{I}$ & 2 & 3 & 4 & 5 & 6 & 7 & 8 & 9 & IO & II & I2 \\
\hline $\mathbf{y}$ & $\mathrm{I}$ & 4 & 9 & $\mathrm{I} 6$ & 25 & 36 & 49 & 64 & $8 \mathrm{I}$ & IOO & I2I & I44 \\
\hline
\end{tabular}

Fonte: elaboração dos autores. 
Figura 8: Linhas de Tendência - Quadrados Perfeitos

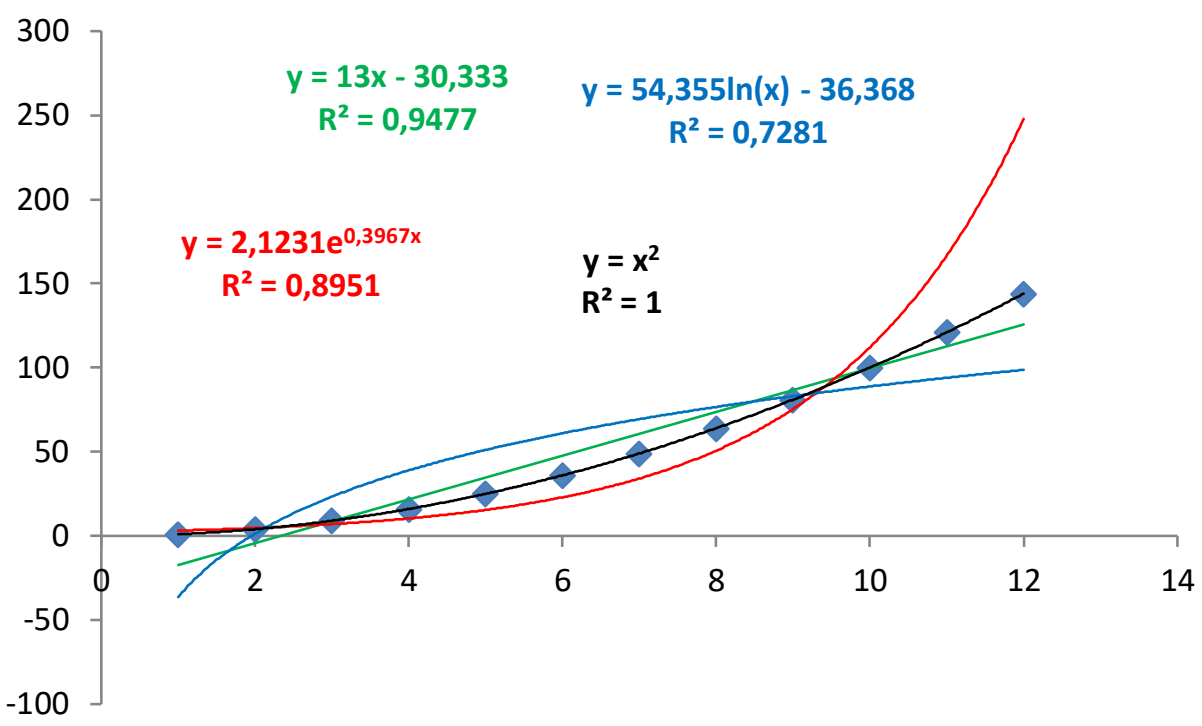

Fonte: elaboração dos autores (Excel).

A Tabela 9 apresenta, para cada sequncia proposta, o melhor e o pior ajuste de regressão para o conjunto de pontos $(x, y)$. Observa-se que com a utilização de uma ferramenta computacional a construção da proposta ficou mais clara e os objetivos foram alcançados.

Tabela 9: Melhor e pior ajuste de regressão, para cada sequência.

\begin{tabular}{ccl}
\hline Sequência & Melhor ajuste & Pior Ajuste \\
\hline Número primo & Potência & Logarítmica \\
Sequência de Fibonacci & Exponencial & Logarítmica \\
Sucessão de Farey & Linear & Exponencial \\
Número hexagonal & Linear & Logarítmica \\
Número de Catalan & Exponencial & Logarítmica \\
PA: $\boldsymbol{a}_{\boldsymbol{n}}=\mathbf{2 n} \mathbf{- 1}$ & Linear & Exponencial \\
PG: $\boldsymbol{a}_{\boldsymbol{n}}=\mathbf{2}^{\boldsymbol{n}}$ & Exponencial & Logarítmica \\
Quadrados perfeitos & Potência & Logarítmica \\
\hline
\end{tabular}

Fonte: elaboração dos autores.

A aquisição de conhecimentos pelo aluno aprendiz é a principal causa e efeito da utilização de um ambiente computacional no processo de ensino e aprendizagem de matemática. As estratégias computacionais de ensino adotada nesta atividade são 
escolhas pedagógicas fundamentais e contribui para o desenvolvimento cognitivo do aluno envolvido.

Nota-se que essa proposta estar recheada de informações associada a uma contextualização entre temas importantes do conteúdo de matemática na educação básica. Sequências numéricas, funções elementares, modelos de regressão e gráficos matemáticos estão interagindo em perfeita sintonia em um ambiente computacional.

Os conteúdos de aprendizagem de matemática, apresentados pelo professor, devem se apresentar para o aluno como desafios de forma a motivá-lo a descobrir novos caminhos. "[...] a matemática deixa de ser um corpo de conhecimentos prontos e simplesmente transmitidos aos alunos e passa a ser algo em que o aluno faz parte integrante no processo de construção de seus conceitos". (D'AMBROSIO, I989, p.5)

Faz-se necessário preconizar aos alunos situações desafiadoras, de tal maneira, que ele possa fazer conjecturas e investigações que o leve a descoberta de novos conhecimentos. Para Ponte (I995) as experiências com o computador tendem a fortalecer uma maior interação e colaboração entre o professor e o aluno. Carneiro e Passos (2014) afirmam que em um ambiente computacional, professor e aluno tornamse parceiros cooperativos, diferente daquela que ocorre em uma sala de aula tradicional, pelo motivo que o aluno, em certo momento, pode ter maior domínio sobre o computador.

O papel do professor deve ser sempre alvo de reflexões, de ter abertura de novas metodologias, de querer melhorar seu redor, romper com paradigmas e certezas de que está tudo bem, sair da condição confortável e tentar encarar o desafio de interpor o novo papel: o de professor mediador, que é trabalhoso, difícil, mas recompensador; o processo não acontece com um único personagem, esse é, sem sombra de dúvidas, o maior desafio do educador matemático (PAULA et al., 2016, p.106)

Assim sendo, a relação professor-aluno deve estar sintonizada, quebrando padrões hierárquicos e regras pré-estabelecidas de quem ensina e de quem aprende. $O$ processo de evolução tecnológica do mundo contemporâneo sugere que o ensino de matemática na educação básica esteja abastecido de novas tecnologias e seus atores, professor e aluno, estejam adaptados com essa transformação.

\section{CONSIDERAÇÕES FINAIS}

O grau de motivação e interesse deste estudo deve-se, basicamente, a interpretação de um modelo matemático usual através de gráficos e equações matemáticas dispondo como instrumento de auxílio um software educativo. Para Pontes (2017, p.2) "Na contemporaneidade é cada vez maior a necessidade de conceber meios na prática educacional de modo que a teoria estudada esteja diretamente correlacionada com modelos usuais dos envolvidos no processo de ensino $e$ aprendizagem".

A transformação tecnológica no mundo moderno exige que a escola esteja preparada para quebrar paradigmas no processo educativo e principalmente na metodologia de ensino e aprendizagem de matemática. $O$ computador e seus softwares 
educativos representam instrumentos metodológicos imprescindíveis no ato de ensinar e no ato de aprender matemática. As aulas de matemática devem ser inovadoras e diversificadas, em que a utilização das parafernálias tecnológicas, em especial o computador, sejam práticas que aproxime o aluno de seu cotidiano.

Portanto, a existência de diferentes modalidades de uso do computador na educação tem o objetivo de atender diferentes interesses educacionais e econômicos. A coexistência destas modalidades é salutar e a decisão por outra modalidade deve levar em consideração a diversidade de variáveis que atuam no processo de ensinoaprendizagem. Se isto for feito, o computador poderá ser um importante aliado desse processo. Caso contrário, não devemos esperar muito dessa tecnologia, pois ela ainda não é capaz de fazer milagres. (VALENTE, 2008, p.15-16)

Diante disto, espera-se que este trabalho possa gerar estímulos necessários para que outros pesquisadores possam buscar práticas que fortaleçam o processo de ensino $e$ aprendizagem de matemática com o propósito de gerar novos conhecimentos e saberes.

\section{REFERÊNCIAS}

ALBUQUERQUE, Marlos Gomes de et al. Um ambiente computacional para aprendizagem matemática baseado no modelo pedagógico de Maria Montessori. 2000.

BRASIL, Parâmetros Curriculares Nacionais para o ensino fundamental: Matemática. Brasília. Ministério da Educação, Secretaria da educação Fundamental e Tecnologia, 1998.

CARNEIRO, Reginaldo Fernando; PASSOS, Cármen Lúcia Brancaglion. A utilização das Tecnologias da Informação e Comunicação nas aulas de Matemática: Limites e possibilidades. Revista Eletrônica de Educação, v. 8, n. 2, p. IOI-II9, 2014.

D’AMBROSIO, Beatriz S. Como ensinar matemática hoje. Temas e debates, v. 2, n. 2, p. 15-19, 1989.

NASCIMENTO, Edvaldo Lopes; SCHIMIGUEL, Juliano. Referenciais teóricos metodológicos: sequenciais didáticas com tecnologias no ensino de matemática na educação básica. Revista de Ensino de Ciências e Matemática, v. 8, n. 2, p. I15-126, 2017.

MISKULIN, Rosana Giaretta Sguerra. As possibilidades didático-pedagógicas de ambientes computacionais na formação colaborativa de professores de matemática. Formação de Professores de Matemática: explorando novos caminhos com outros olhares. Campinas: Mercado das Letras, p. 217-248, 2003. 
NASCIMENTO, Edvaldo Lopes; SCHIMIGUEL, Juliano. Referenciais teóricos metodológicos: sequenciais didáticas com tecnologias no ensino de matemática na educação básica. Revista de Ensino de Ciências e Matemática, v. 8, n. 2, p. II5-126, 2017. PAULA, Samantha Chang Rodrigues de. Educação matemática e tecnologia: articulando práticas geométricas/ Samantha Chang Rodrigues de Paula, Chang Kuo Rodrigues, Julio César da Silva. I. ed. Curitiba: Appris, 2016.

PONTE, João Pedro da. Novas tecnologias na aula de Matemática. Educação e Matemática, p. 2-7, 1995. .

PONTES, Edel Alexandre Silva. HIPERMAT-Hipertexto Matemático: Uma ferramenta no ensino-aprendizagem da matemática na educação básica. Psicologia \& Saberes, v. 2, n. 2, 2013.

PONTES, Edel Alexandre Silva et al. Raciocínio lógico matemático no desenvolvimento do intelecto de crianças através das operações adição e subtração. Diversitas Journal , 2 v. 3 n. p. $469-476.2017$.

PONTES, Edel Alexandre Silva. O uso de uma prova paramétrica para tomada de decisão em uma pesquisa de campo: um eficiente processo de ensino e aprendizagem. Revista Educação \& Tecnologia, v. 17, p. I-I0.2017, 2017.

PONTES, Edel Alexandre Silva et al. O estudo do comportamento da sucessão de Fibonacci através de diversas linhas de tendência associadas as suas respectivas equações matemáticas de regressão. Diversitas Journal, v. 3, n. 3, p. 771-779, 2018.

PONTES, Edel Alexandre Silva. Método de polya para resolução de problemas matemáticos: uma proposta metodológica para o ensino e aprendizagem de matemática na educação básica. HOLOS, v. 3, p. I-9, 2019.

PONTES, Edel Alexandre Silva et al. Prática educacional no ato de ensinar e aprender matemática nos anos finais do ensino fundamental por meio do processoRICA: Raciocínio lógico, Inteligência matemática, Criatividade e Aprendizagem Educational practice in the act of teaching and learning mathematics in the final years of elementary school through the process-RICA. Brazilian Applied Science Review, v. 5, n. 3, p. I4II-I424, 2021.

TENÓRIO, André; DO NASCIMENTO, Carla P. V.; TENÓRIO, Thais. Uso do software Geogebra, blog, e-mail e whatsapp no estudo de Matemática. Revista Tecnologias na Educação, I9 v. 8 n. 2017.

VALENTE, José Armando. Diferentes usos do computador na educação. Em Aberto, v. 12, n. 57, 2008. 